\title{
Validation of the Publication of New Names and New Combinations Previously Effectively Published Outside the IJSB
}

\section{List No. $2 \dagger$}

The purpose of this announcement is to effect the valid publication of the following new names and new combinations under the procedure described previously [Int. J. Syst. Bacteriol. 27(3):iv, 1977]. Authors and other individuals wishing to have new names and/or combinations included in future lists should send the pertinent reprint(s) to the Editor of the IJSB for confirmation that all of the other requirements for valid publication have been met. It should be noted that the date of valid publication of these new names and combinations is the date of publication of this list, not the date of the original publication of the names and combinations. The authors of the new names and combinations are as given below, and these authors' names will be included in the Author Index of the present issue and in the volume Author Index in the October issue of the IJSB.

\section{LITERATURE CITED}

1. Adams, M. M., J. N. Adams, and G. H. Brownell. 1970. The identification of Jensenia canicruria Bisset and Moore as a mating type of Nocardia erythropolis (Gray and Thornton) Waksman and Henrici. Int. J. Syst. Bacteriol. 20:133-147.

2. Barnes, E. M., C. S. Impey, B. J. H. Stevens, and J. L. Peel. 1977. Streptococcus pleomorphus sp. nov.: an anaerobic streptococcus isolated mainly from the caeca of birds. J. Gen. Microbiol. 102:45-53.

3. Beijerinck, M. W. 1925. Über ein Spirillum, welches freien Stickstoff binden kann? Zentralbl. Bakteriol. Parasitenkd. Infektionskr. Hyg. Abt. 2 63:353-359.

4. Goodfellow, M., and G. Alderson. 1977. The actinomycete-genus Rhodococcus: a home for the "rhodochrous' complex. J. Gen. Microbiol. 100:99-122.

5. Gray, P. H. H., and H. G. Thornton. 1928. Soil bacteria that decompose certain aromatic compounds. Zentralbl. Bakteriol. Parasitenkd. Infektionskr. Hyg. Abt. 2 73: 74-96.

6. Imhoff, J. F., and H. G. Trüper. 1977. Ectothiorhodo-

$\dagger$ List number 1 was published in: Int. J. Syst. Bacteriol. 27:306, 1977 . spira halochloris sp. nov., a new extremely halophilic phototrophic bacterium containing bacteriochlorophyll b. Arch. Microbiol. 114:115-121.

7. Kuhn, D. A., and D. A. Gregory. 1978. Emendation of Simonsiella muelleri Schmid and description of Simon. siella steedae sp. nov., with designations of the respective proposed neotype and holotype strains. Curr. Microbiol. 1:11-14.

8. Reichelt, J. L., P. Baumann, and L. Baumann. 1976. Study of genetic relationships among marine species of the genera Beneckea and Photobacterium by means of in vitro DNA/DNA hybridization. Arch. Microbiol. 110:101-120.

9. Rowbotham, T. J., and T. Cross. 1977. Rhodococcus coprophilus sp. nov.: an aerobic nocardioform actinomycete belonging to the 'rhodochrous' complex. J. Gen. Microbiol. 100:123-138.

10. Tarrand, J. J., N. R. Krieg, and J. Döbereiner. 1978. A taxonomic study of the Spirillum lipoferum group, with descriptions of a new genus, Azospirillum gen. nov. and two species, Azospirillum lipoferum (Beijerinck) comb. nov. and Azospirillum brasilense sp. nov. Can. J. Microbiol. 24:967-980.

11. Trejo, W., L. D. Dean, J. Pluscec, E. Meyers, and W. E. Brown. 1977. Streptomyces laurentii, a new species producing thiostrepton. J. Antibiot. 30:639-643. 


\begin{tabular}{|c|c|c|c|c|c|}
\hline \multirow[b]{2}{*}{ Name or combination } & \multirow[b]{2}{*}{ Proposed as: } & \multirow[b]{2}{*}{ Author(s) } & \multicolumn{2}{|c|}{$\begin{array}{l}\text { Literature cita- } \\
\text { tion(s) to: }\end{array}$} & \multirow[b]{2}{*}{ Nomenclatural type $^{a}$} \\
\hline & & & $\begin{array}{c}\text { Pro- } \\
\text { posal of } \\
\text { name or } \\
\text { combi- } \\
\text { nation }\end{array}$ & $\begin{array}{l}\text { De- } \\
\text { scrip- } \\
\text { tion of } \\
\text { the } \\
\text { taxon }\end{array}$ & \\
\hline Azospirillum & New genus & Tarrand et al. & 10 & 10 & $\begin{array}{l}\text { A. lipoferum (Beijerinck) } \\
\text { Tarrand et al. }{ }^{b}\end{array}$ \\
\hline $\begin{array}{l}\text { Azospirillum brasi. } \\
\text { lense }\end{array}$ & New species & Tarrand et al. & 10 & 10 & $\begin{array}{c}\text { Strain sp. } 7(=\text { ATCC } \\
29145)^{b}\end{array}$ \\
\hline $\begin{array}{l}\text { Azospirillum lipo- } \\
\text { ferum }\end{array}$ & New combination & $\begin{array}{l}\text { (Beijerinck) Tarrand } \\
\text { et al. }\end{array}$ & 10 & 3,10 & $\begin{array}{l}\text { Strain sp. 59b }(=\text { ATCC } \\
29707)^{c}\end{array}$ \\
\hline Beneckea vulnifica & New species & Reichelt et al. & 8 & 8 & $\underset{27562)^{b}}{\text { Strain } 324} \quad(=$ ATCC \\
\hline $\begin{array}{l}\text { Ectothiorhodospira } \\
\text { halochloris }\end{array}$ & New species & Imhoff and Trüper & 6 & 6 & $\begin{array}{l}\text { Strain BN } 9850(=\text { DSM } \\
\text { 1059) }^{b}\end{array}$ \\
\hline $\begin{array}{l}\text { Photobacterium an- } \\
\text { gustum }\end{array}$ & New species & Reichelt et al. & 8 & 8 & $\begin{array}{l}\text { Strain } \\
\left.2_{25915}\right)^{b}\end{array}$ \\
\hline $\begin{array}{l}\text { Rhodococcus copro- } \\
\text { philus }\end{array}$ & New species & $\begin{array}{l}\text { Rowbotham and } \\
\text { Cross }\end{array}$ & 9 & 9 & $\begin{array}{l}\text { Strain CUB } 687 \quad(= \\
\text { ATCC } 29080=\text { NCIB } \\
11211=\text { NCTC } 10994)^{b}\end{array}$ \\
\hline $\begin{array}{l}\text { Rhodococcus erythro- } \\
\text { polis }\end{array}$ & New combination & $\begin{array}{l}\text { (Gray and } \\
\text { Thornton) Good- } \\
\text { fellow and Alder- } \\
\text { son }\end{array}$ & 4 & 4,5 & $\begin{array}{c}\text { Strain N11 }(=\text { ATCC } \\
4277=\text { NCIB 9158 })^{d}\end{array}$ \\
\hline Rhodococcus rhodnii & New species & $\begin{array}{l}\text { Goodfellow and Ald- } \\
\text { erson }\end{array}$ & 4 & 4 & $\underset{11279)^{b}}{\text { Strain N445 }} \quad(=$ NCIB \\
\hline Simonsiella steedae & New species & Kuhn and Gregory & 7 & 7 & $\begin{array}{l}\text { Strain ATCC } 27409(= \\
\text { ICPB 3604) })^{b}\end{array}$ \\
\hline $\begin{array}{l}\text { Streptococcus pleo- } \\
\text { morphus }\end{array}$ & New species & Barnes et al. & 2 & 2 & $\begin{array}{l}\text { Strain EBF } 61 / 60 B(= \\
\text { NCTC } 11087)^{b}\end{array}$ \\
\hline Streptomyces laurentii & New species & Trejo et al. & 11 & 11 & Strain ATCC $31255^{b}$ \\
\hline
\end{tabular}

a Abbreviations: ATCC, American Type Culture Collection, Rockville, Md.; DSM, Deutsche Sammlung von Mikroorganismen, Göttingen, Germany; ICPB, International Collection of Phytopathogenic Bacteria, University of California, Davis, Calif.; NCIB, National Collection of Industrial Bacteria, Aberdeen, Scotland; NCTC, National Collection of Type Cultures, London, England.

${ }^{b}$ Type designated by the author(s).

${ }^{c}$ Proposed neotype. A proposed neotype becomes established 2 years after the date of the publication of the proposal in the IJSB, providing that there are no objections, which must be referred to the Judicial Commission of the International Committee on Systematic Bacteriology within the first year after the proposal of the neotype.

$d$ Type strain designated in reference 1. 